\section{FORTALECIENDO EL USO DE LOS INSTRUMENTOS DE PLANIFICACIÓN URBANA, PARA LA GESTIÓN DE RIESGOS. CIUDAD DE CORONEL, REGIÓN DEL BIOBÍ0*}

Edilia del Carmen Jaque Castillo ${ }^{\alpha}$

Alejandro Lara San Martin ${ }^{\beta}$

Camila Merino González ${ }^{\gamma}$

\section{Resumen}

La migración hacia asentamientos urbanos espontáneos, en zonas expuestas a amenazas naturales en los países en desarrollo, aumenta la vulnerabilidad de la población frente a desastres socionaturales. En Chile se ha incorporado la variable de riesgos en los instrumentos de planificación territorial, a través de estudios que se integran a la propuesta de zonificación de usos de suelo. A objeto de promover el uso de la información generada en la gestión de los riesgos por parte de las instituciones ligadas al manejo de desastres, se empleó la zonificación de amenazas de la ciudad de Coronel, Región del Biobío, a través del análisis

\section{STRENGTHENING THE USE OF URBAN PLANNING INSTRUMENTS FOR RISK MANAGEMENT PURPOSES. THE CITY OF CORONEL, BIOBÍO REGION*}

\author{
Edilia del Carmen Jaque Castillo ${ }^{\alpha}$ \\ Alejandro Lara San Martin ${ }^{\beta}$ \\ Camila Merino González ${ }^{\gamma}$
}

\section{Abstract}

In developing countries, the migration to unplanned urban settlements located in areas exposed to natural hazards has increased the vulnerability of the population to socionatural disasters. In Chile, the variable of risk has been included in territorial planning instruments through studies on land use zoning. In order to promote the use of the information generated by risk management initiatives, the authorities of Coronel identified risk areas through spatial analysis intended to determine the distribution and exposure of local dwellers. The results show that 67.2 percent of urban land is prone 
espacial para determinar la distribución y exposición de sus habitantes. Los resultados indicaron que el $67,2 \%$ de la superficie urbana de Coronel está expuesta a diversas amenazas socionaturales, las cuales afectan al 18,4\% de su población.

\section{PALABRAS CLAVE: RIESGO, GESTIÓN DEL RIESGO, PLANIFICACIÓN URBANA, PLAN REGULADOR COMUNAL.}

\author{
Recibido: 25/06/2015 \\ Aceptado: 04/10/2016
}

* Este artículo fue elaborado a partir de la investigación “Valoración de los riesgos naturales en un territorio socioeconómicamente degradado, provincia de Arauco, Región del Biobío: orientaciones para la gestión integrada del territorio". DIUC FI nro. 209.603.010-1. 208.603.007-1.0, Vicerrectoría de Investigación, Universidad de Concepción.

$\alpha \quad$ Chile. Departamento de Geografía, Facultad de Arquitectura, Urbanismo y Geografía, Universidad de Concepción. Correo electrónico: edjaque@udec.cl.

$\beta \quad$ Chile. Departamento de Arquitectura, Universidad de Concepción. Correo electrónico: alejandrolara@udec.cl.

$\gamma \quad$ Chile. Secretaria Comunal de Planificación, Municipalidad de Concepción. Correo electrónico: merinocamila@gmail.com. to socionatural disasters, which may affect 18.4 percent of the local population.

KEYWORDS: RISK, RISK MANAGEMENT, URBAN PLANNING, MUNICIPAL MASTER PLAN.

Received: 25/06/2015

Accepted: 04/10/2016

* This paper is part of the research project "Valuation of Natural Hazards in a Socioeconomically Degraded Territory, Province of Arauco, Biobío Region: Guidelines for Territory-Related Management". DIUC FI No. 209.603.010-1. 208.603.007-1.0, Research Department, University of Concepción.

$\alpha \quad$ Chile. Department of Geography, Faculty of Architecture, Urban Planning and Geography, University of Concepción. Email: edjaque@udec.cl.

$\beta \quad$ Chile. Department of Architecture, University of Concepción. Email: alejandrolara@udec.cl.

$\gamma \quad$ Chile. Municipal Planning Department, Municipality of Concepción.Email:merinocamila@gmail.com. 


\section{Introducción}

La trascendental importancia de estudiar y comprender el territorio, es que toda actividad humana está inherentemente ligada a él. Es aquí donde las normas y políticas cobran vida para la consecución de un fin ulterior, como lo es el bien común, el cual estaría vinculado al concepto de desarrollo. Cuando nos referimos al desarrollo, involuntariamente surgen a nuestros pensamientos nombres como Adam Smith, David Ricardo o Carlos Marx, cuyas definiciones de este complejo concepto estaban muy ligadas a concepciones económicas. No obstante, el desarrollo es uno de los conceptos más poderosos de nuestra época, pues implica la toma de decisiones entre un conjunto de opciones acerca de metas que reflejen lo que debería ser una sociedad óptima (Mendoza, 2000). En la actualidad, el concepto desarrollo no podemos entenderlo como un concepto aislado, sino más bien asociado a ciertos objetivos que, en un momento determinado, la sociedad busca concretar y cuyas acciones se visualizan en un espacio geográfico, entendido como territorio (Boisier, 2001).

De lo anterior se desprenden dos conceptos claves vinculados al desarrollo del territorio, como la planificación urbana y el ordenamiento territorial. Silva y Romero (2015), indican que el planeamiento urbano tiene un carácter globalizador como instrumento de desarrollo urbano en su sentido más amplio, pero no deja de ser un instrumento normativo cuya función básica es clasificar y calificar el suelo, y garantizar unas dotaciones adecuadas de servicios, infraestructuras y equipamiento comunitario. Lo anterior permite, de acuerdo a Muñiz, Sánchez y García (2015), una elevada calidad de vida de la población residente, como el desarrollo eficiente de las actividades económicas que se localizan en el espacio. Por otro lado, la ordenación territorial se comprende como un intento de integrar la planificación socioeconómica con la física, procurando la consecución de la estructura espacial adecuada para un desarrollo eficaz y equitativo de la política económica, social, cultural y ambiental de la sociedad, para así avanzar hacia el sistema territorial de largo plazo (Gómez, 2012; SUBDERE, 2011; Pujadas y Font, 1998).

Sin embargo, encontramos en la práctica que la ejecución de estos dos conceptos unidos al de desarrollo, no necesariamente están integrados, ni reflejan un enfoque basado en el consenso. Uno de los principales efectos de la intervención del ser humano en el medio ambiente es la transformación de espacios no aptos para la urbanización, la cual se basa en la idea de desarrollo, en centros poblados y de servicios (Serra, 2011). Estudios sobre migraciones indican que la proporción de la población urbana mundial pasó de representar el 13\% de la población total en 1900 al $49 \%$ en 2005 y al 51,3\% en 2010. Es probable que esta cifra 
alcance el 57\% en 2025 y llegue cerca del $70 \%$ en 2050 (Burgos y Muñoz, 2007), ya que en Chile alcanza el 86,5\% de acuerdo al censo del año 2002. Esto genera una mayor presión tanto en el medio ambiente como en el uso del suelo (Martí, 2005) y en la ocupación de terrenos que están expuestos a diversas amenazas naturales.

Cada año vemos cómo los riesgos se transforman en desastres, provocando muertes, desaparecidos, heridos, evacuados y desplazados, produciendo daños graves a la propiedad y al medio natural (United Nations International Strategy for Disaster Reduction, 2009). Es por ello que, para entender de manera adecuada la problemática de los riesgos, se requiere de una comprensión de la naturaleza y la magnitud de los mismos, es decir, de los procesos que los generan, la probabilidad de que ocurran y sus posibles consecuencias (Bevilacqua, Ciarapica y Paciarotti, 2014). Los riesgos naturales son acontecimientos naturales extremos, percibidos por el hombre como una amenaza para su vida y para sus bienes. La ocurrencia del riesgo conduce a un evento extremo, en un lugar y tiempo determinado, que puede culminar en un desastre. Así los riesgos naturales están directamente relacionados con el hombre y con las alteraciones que él mismo causa al instalarse en el medio, pues la ciudad, invención humana construida sobre el sistema natural, provoca desequilibrios en este, ya que modifica gravemente sus procesos.
La idea de los límites del crecimiento retomada por Beck (2002), hace ver las contradicciones de la sociedad contemporánea, señalando que los riesgos son en gran medida derivados de la propia intervención del ser humano en el planeta, particularmente las derivadas del sistema técnico-científico. De esta forma, una de las dimensiones de la crisis antes señalada la constituye la construcción social del riesgo. Este enfoque hace referencia a los procesos a través de los cuales la sociedad y los distintos agentes sociales contribuyen a la creación de contextos y entornos de riesgo. En este sentido, se comprende que el territorio es un concepto más flexible, no solo representa el soporte geopolítico de los estados nacionales, sino que dicho concepto constituye una manifestación más versátil del espacio social, como reproductor de las acciones de los actores sociales (Llanos-Hernández, 2013). Concepción que podemos materializar en palabras de Deleuze y Guattari

"nada se piensa, se hace, ni se cambia si no es a través de la materialidad de los lugares y de sus propiedades, porque es a través de estas -de las cosas ligadas al suelo-que pasan necesariamente (aunque no de un modo determinista) todas las relaciones sociales y sus representaciones conceptuales" (1999, p. 86).

Hoy comprendemos que los riesgos se desarrollan en un entorno complejo y dinámico, es decir, el escenario donde se observan estos fenómenos 
es impredecible, incierto e incontrolable (Ashly, 2010), donde además de la variabilidad del clima y el cambio climático, se hace necesaria una revisión de las estrategias de desarrollo y gestión del riesgo a largo plazo (Zilbert, 2010; Klijn, van Buuren y van Rooij, 2004). La introducción de los riesgos naturales en la planificación urbana de Chile, comienza con la Ordenanza General de Urbanismo y Construcción de 1992, la cual señala que en los futuros planes reguladores se deben identificar áreas de riesgo y las acciones realizadas por la planificación y el ordenamiento territorial, se deben considerar medidas de prevención ante los riesgos naturales. En este contexto, el objetivo de esta investigación se centra en conocer la exposición de la población urbana de Coronel, a los distintos tipos de amenazas naturales, incluidas en el instrumento de planificación, más allá de la zonificación de usos de suelo. Lo anterior se empleó como base de datos para diagnosticar el estado actual de la población expuesta a amenazas naturales en la ciudad, ofreciendo herramientas espaciales de análisis a los organismos encargados de comandar los distintos comités de emergencia.

Chile, post terremoto de 1939, en lo referido a su enfoque de desarrollo territorial, adopta el modelo de desarrollo de polos, donde se hace propio el concepto de producción masiva de bienes y servicios, promovido por la creación de la Corporación de Fomento de la Producción (CORFO). Este organismo, facilitó un proceso de industrialización de zonas específicas del país, entre ellas la actual provincia de Concepción (Aliste, Contreras y Sandoval, 2012).

El impulso industrial de la década del cuarenta, trajo consigo el inicio de la instalación de complejos industriales en la zona, hecho que podría considerarse como el principal responsable del proceso de migración rural-urbano, motivado por la nueva fuerza laboral que requería el desarrollo de la industria. Esto llevó consigo una expansión significativa del área urbana, hacia localidades comunales más alejadas, produciéndose fenómenos de expansión desenfrenada, fomentando así una presión sobre los territorios, el medio ambiente y el uso del suelo (Aliste et al., 2012; Fuentes y Pérez, 2012). Especialmente en aquellos espacios que se emplazan en llanuras litorales y fluviales, desembocaduras de ríos y esteros, con un entorno topográficamente abrupto y altamente erosionado, donde las condiciones geográficas físicas y de accionar humano, aumentan la probabilidad de ocurrencia de fenómenos de riesgos como inundaciones, marejadas, anegamiento, deslizamientos de tierra y tsunamis (Rojas, Muñiz y Pino, 2013). Además de generar disyuntivas territoriales y conflictos ambientales muy fuertes, al ubicar poblaciones cerca de instalaciones industriales (Smith y Romero, 2009; Suazo, Jaque, Rojas y Utz, 2009). 


\section{FIGURA 1. LOCALIZACIÓN DE LA CIUDAD DE CORONEL}

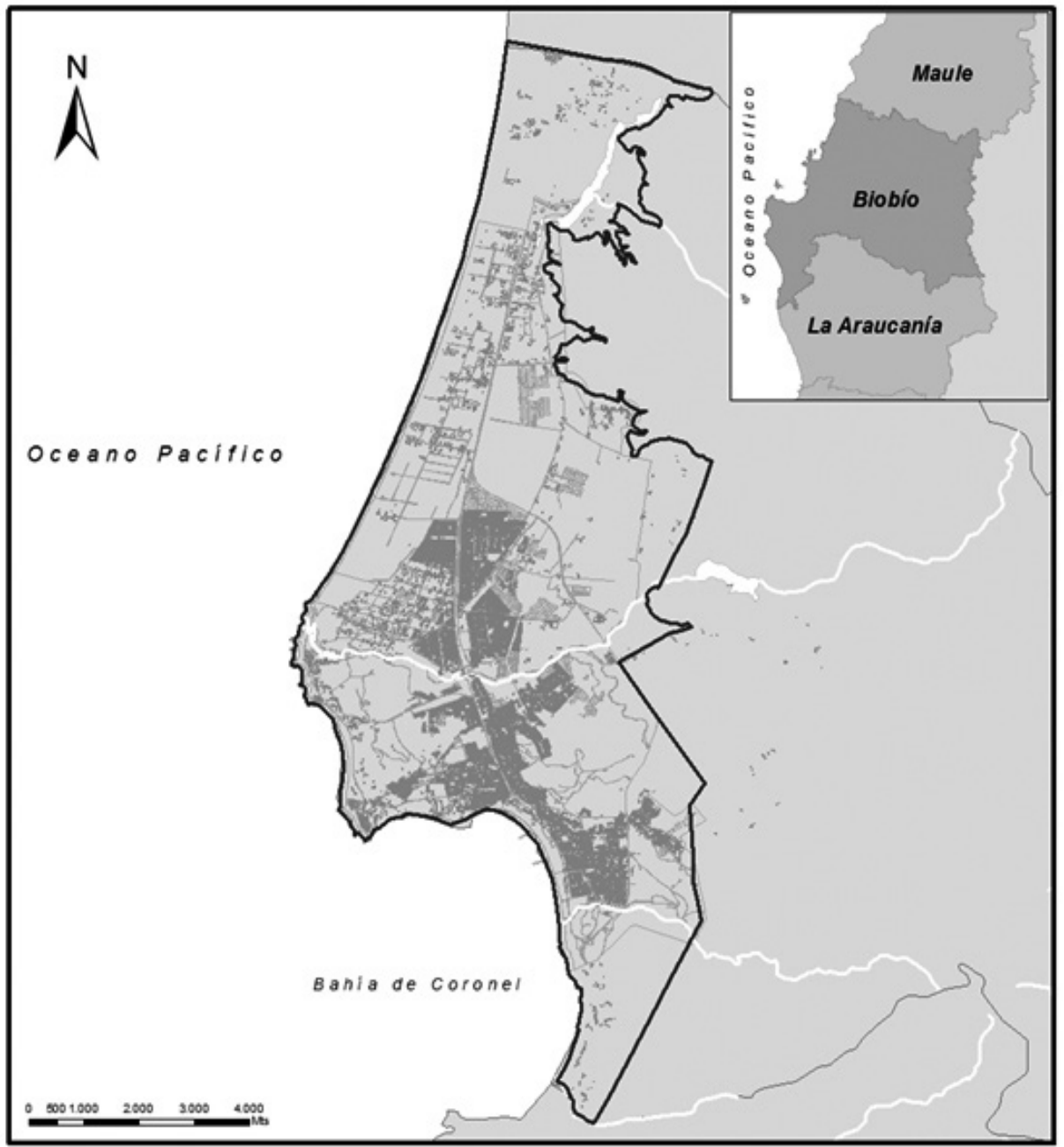

Fuente: Elaboración propia. 


\section{TABLA 1. SUPERFICIE AFECTADA POR TIPOS DE AMENAZA NATURAL EN LA CIUDAD DE CORONEL}

\begin{tabular}{ll} 
Amenazas & Superficie en $\mathrm{km}^{2}$ \\
\hline Anegamiento & 9,88 \\
Subsidencia & 1,87 \\
Remoción en Masa & 7,01 \\
\hline Tsunami & 12,23
\end{tabular}

Fuente: Elaboración propia, a partir de la carta de amenazas naturales.

La ciudad de Coronel localizada en la vertiente occidental del macizo de Nahuelbuta ( $36^{\circ} 58^{\prime}$ S y $73^{\circ} \mathrm{O}$-figura 1-), es la tercera comuna más poblada del área metropolitana de Concepción, con una población urbana de 91.469 habitantes, según datos de censo de 2002. Desde 1970 su población total ha aumentado con una tasa de crecimiento anual promedio de 1,53\%, (figura 2), incrementándose en un 62\% hasta el año 2002, su población rural ha crecido en 3\% y su población urbana en un $66,9 \%$, esta es considerablemente superior a la rural siendo el 95,8\% de la población total en el año 2002. Este incremento de la población, ha implicado la saturación del área urbana expandiéndose fuera del límite urbano existente, así se han ido configurando nuevos territorios en riesgo generando, de esta manera, una mayor exposición de la población a diversas amenazas naturales. Entendidas estas como procesos o fenómenos de carácter natural o tecnológico, que pueden originar daños a la población, a los bienes materiales o al medio ambiente (Ayala y Olcina, 2002), es también la probabilidad de que ocurra un fenómeno frente al cual esa comunidad particular es vulnerable (Wilches-Chaux, 1993).

La superficie afectada por amenaza de remoción en masa (tabla 1), alcanza una superficie de 7,01 $\mathrm{km}^{2}$ en el área urbana. La amenaza de subsidencia alcanza una superficie de $1,87 \mathrm{~km}^{2}$, se hace parte de la ciudad de Coronel desde el inicio de la actividad minera carbonífera, a raíz de esto, su casco urbano se encuentra poblado de túneles de extracción del mineral de carbón denominados pirquenes, que están en su mayoría abandonados.

La amenaza por anegamiento, afecta a una superficie de $9,88 \mathrm{~km}^{2}$ del área urbana, estos se producen principalmente debido a la saturación de las napas en suelos anegadizos, a la insuficiencia de los sistemas de drenaje y a la impermeabilidad generada por las coberturas urbanas. Los distintos temporales que ocurren en la región y particularmente en la provincia de Concepción, propician estos episodios de inundaciones en Coronel, tal fue el caso del temporal ocurrido en el año 2006, que dejó 225 damnificados y 24 viviendas arruinadas, correspondiendo al $6,6 \%$ de las viviendas destruidas en la provincia (Onemi, 2006). La amenaza por inundación de tsunami corresponde a una superficie de $12,23 \mathrm{~km}^{2}$. Y se debe al emplazamiento costero de la ciudad. 


\section{FIGURA 2. CRECIMIENTO URBANO DE LA COMUNA DE CORONEL ENTRE 1970 - 2002}

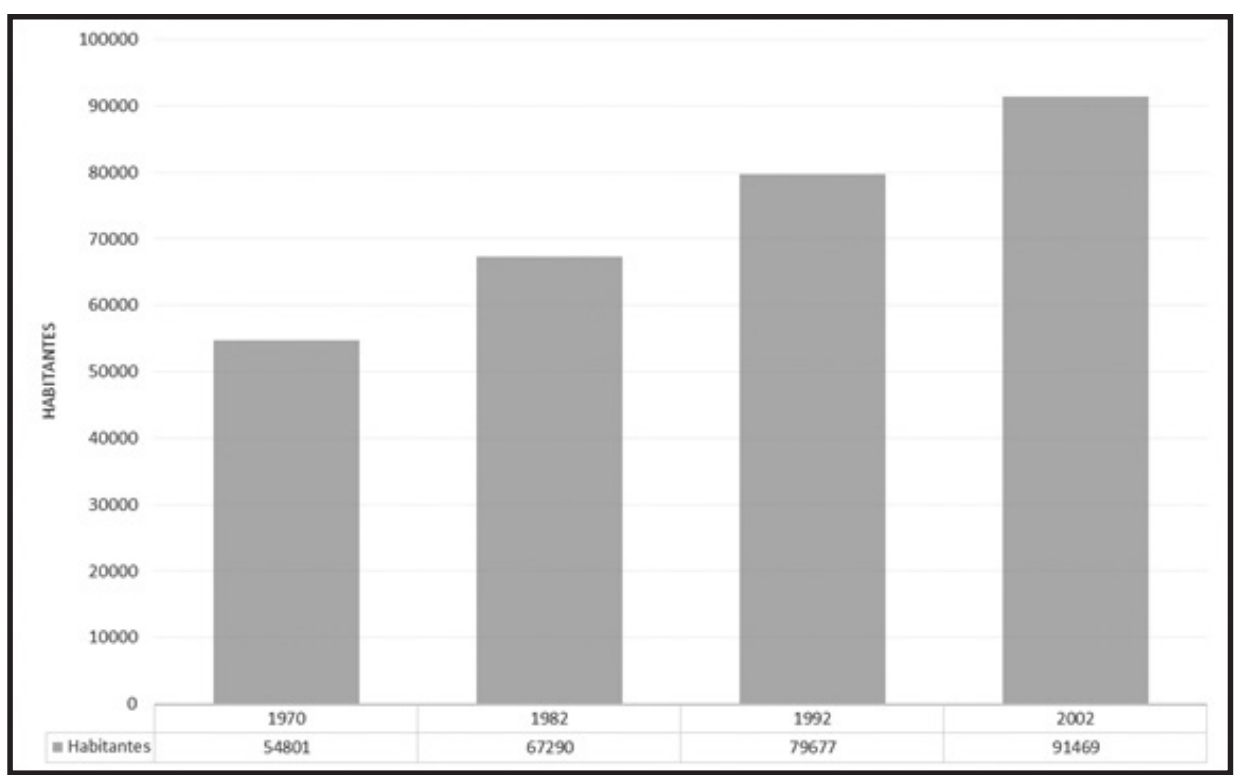

Fuente: INE 2005.

\section{Metodología}

La técnica utilizada, se basa en la modelación de los riesgos en Sistema de Información Geográfica (SIG) y en el cálculo de su exposición mediante análisis de superposición espacial de mapas de amenaza. Para generar la cartografía de amenazas de Coronel, se utilizó la carta vectorial de riesgos naturales escala 1:100.000, generada por el estudio fundado del Plan Regulador Comunal de Coronel del año 2004. Esta cartografía se desarrolló bajo la premisa que es el proceso o fenómeno de carácter natural o tecnológico que puede originar daños a la población, a los bienes materiales o al medio ambiente (Ayala y Olcina, 2002).

Para elaborar la cartografía de exposición, se utilizó la unidad de manzanas censales del año 2002, en formato vectorial escala 1:10.000 y la base de datos asociada al censo de población y vivienda 
FIGURA 3. CARTOGRAFÍA DE AMENAZAS NATURALES ÁREA URBANA DE CORONEL

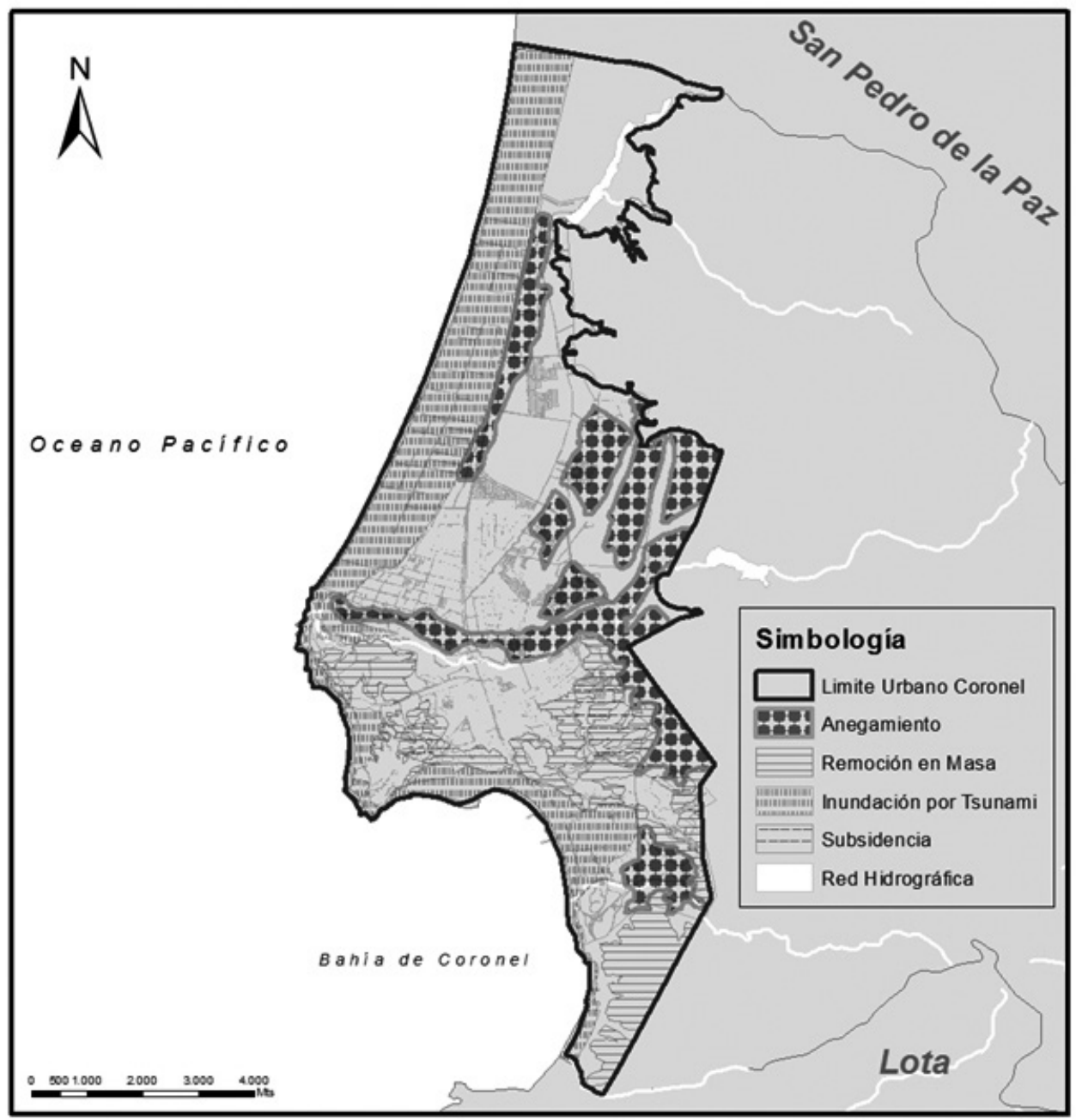

Fuente: Elaboración propia a partir del PRC de Coronel, 2004. 
FIGURA 4. CARTA DE EXPOSICIÓN A AMENAZAS NATURALES A) AMENAZA POR ANEGAMIENTO; B) AMENAZA POR REMOCIÓN EN MASA; C) AMENAZA POR SUBSIDENCIA Y D) AMENAZA POR INUNDACIÓN DE TSUNAMI EN LA CIUDAD DE CORONEL

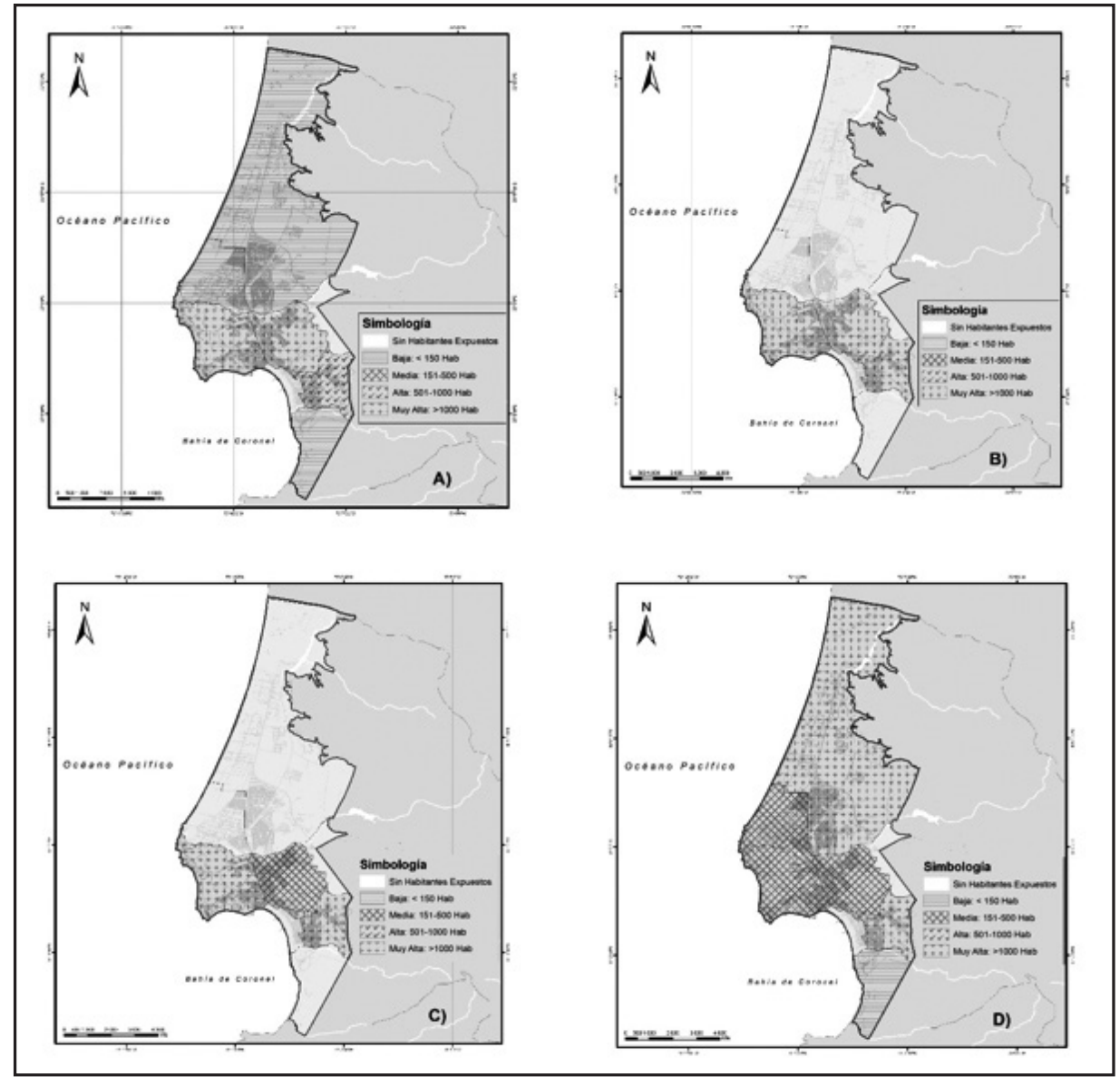

Fuente: Elaboración propia. 
del mismo año. La variable población se obtuvo mediante consultas en el software Redatam+SP (C) del Censo año 2002, cuya tabla de atributos se unió con la información espacial en el software ArcGIS 9.3. Esta cartografía se desarrolló bajo la premisa que la exposición es la disposición sobre el territorio de un conjunto de bienes a preservar que pueden ser dañados por una amenaza natural (UNISDR, 2004, p. 15), incorporando en este concepto a las personas que habitan la zona amenazada. Para clasificar la población según los tipos de riesgo, se realizaron geoprocesos de superposición, mediante el cual se identificaron las áreas de mayor exposición al riesgo.

\section{Resultados}

\section{LA EXPOSICIÓN FRENTE A AMENAZAS EN LA CIUDAD DE CORONEL}

Los procesos naturales que identificamos como amenazas dentro del límite urbano actual de Coronel son: inundación por tsunami, anegamiento, subsidencia y remoción en masa. La amenaza que presenta una mayor exposición de población es la de remoción en masa con 11.354 personas. A la amenaza por tsunami están expuestas 5.208 personas. En el caso de la amenaza por subsidencia son 5.080 las personas que se verían afectadas, de las que 4.205 están, de igual modo, expuestas a procesos de remoción en masa. La amenaza que presenta un menor número de personas expuestas, es el anegamiento con 4.633 habitantes.

Analizado desde la perspectiva de la exposición a las amenazas por distrito censal, se constató que donde existe la mayor cantidad de población expuesta a algún tipo de amenaza es en el distrito censal de Schwager, con el 53,1\%, equivalente a 8.322 personas expuestas. Villa Mora tiene un $37,3 \%$ de su población expuesta, es decir 5.637 personas habitando en zonas con amenazas naturales. El distrito censal de Corcovado tiene un 31,6\% de su población expuesta, con 2.650 habitantes. Los distritos con menor población expuesta a amenazas son: Buen Retiro con un 0,73\%, con 118 personas, seguida por Escuadrón con 0,34\%, con 107 personas, y Playa Negra, donde la totalidad de sus habitantes se encuentran expuestos a la amenaza de anegamiento (tabla 2). 
TABLA 2. POBLACIÓN EXPUESTA A AMENAZAS NATURALES SEGÚN DISTRITO CENSAL

\begin{tabular}{|lrrrr}
\hline $\begin{array}{l}\text { Código de distrito } \\
\text { censal }\end{array}$ & Distrito censal & Población & $\begin{array}{r}\text { Población expuesta en } \\
\text { nro. de habitantes }\end{array}$ & $\begin{array}{r}\text { Población expuesta en } \\
\text { porcentaje (\%) }\end{array}$ \\
\hline 1 & Municipalidad & 4.883 & 0 & 0 \\
\hline 2 & Villa mora & 15.090 & 5.637 & 37,3 \\
\hline 3 & Schwager & 15.655 & 8.322 & 53,1 \\
\hline 4 & Buen retiro & 15.995 & 118 & 0,73 \\
\hline 8 & Corcovado & 8.367 & 2.650 & 31,6 \\
\hline 9 & Playa negra & 28 & 28 & 100 \\
\hline 11 & Escuadrón & 31.398 & 107 & 0,34 \\
\hline Total & & 91.416 & 16.862 & \\
\hline
\end{tabular}

Fuente: Elaboración propia, a partir de los análisis de los autores de la carta de amenazas y datos del Instituto Nacional de Estadísticas (INE).

\section{EXPOSICIÓN FRENTE A LA AMENAZA DE PROCESOS DE REMOCIÓN EN MASA}

La amenaza por procesos de remoción en masa, definida como procesos en que se desplazan grandes volúmenes de material en las laderas, a veces de carácter catastrófico (Jaque, 1995); es el fenómeno natural que presenta mayor número de personas expuestas con 11.354 equivalente al 29\%, las que se sitúan en tres distritos censales: Schwager, Villa Mora y Corcovado.

En el distrito censal de Schwager hay 5.638 habitantes expuestos, equivalentes al $36 \%$ de la población, estos están distribuidos en el sector de Maule
(285 personas) y en Cerro Obligado (5.353 personas), las que se concentran principalmente hacia el sector de la bahía, en las poblaciones: La colonia, Puchoco y Cerro Obligado. En el distrito censal de Villa Mora 4.031 personas se sitúan en áreas expuestas, equivalentes al 26,7\%, las que están casi en su totalidad ubicadas en el cerro Merquín. En el distrito de Corcovado son 1.685 personas, que representan al 20,1\% expuesto a esta amenaza y se localizan en el sector cerro La Virgen, cercano al centro cívico de la ciudad (652 personas), en cerro 
Corcovado II (458 personas), y cerro Corcovado I (575 personas).

\section{EXPOSICIÓN A LA AMENAZA DE SUBSIDENCIA}

La amenaza por fenómenos de subsidencia, entendida como el hundimiento paulatino del suelo, originado por las cavidades subterráneas producidas por las extracciones mineras (Quezada, Jaque, Fernández y Vásquez, 2012), afecta a una población de 5.080 personas, representando al 12,9\%, y se manifiesta en los distritos de Villa Mora y Corcovado, los cuales están afectos al proceso de remoción en masa.

En el distrito de Schwager, 3.699 personas están ubicadas en áreas expuestas, lo que corresponde al 23,6\% de la población, de las cuales 198 personas se distribuyen en la localidad de Maule y 3.501 en cerro Obligado. En este distrito, 2.956 personas se encuentran amenazadas por procesos de remoción en masa. En Villa Mora, 240 personas están expuestas, equivalentes al 1,5\%, concentradas en cerro Merquín, quienes además están expuestas a procesos de remoción en masa. Finalmente en el distrito censal de Corcovado encontramos 1.141 habitantes expuestos, los cuales representan al $13,6 \%$, de los cuales 1.009 se encuentran en áreas afectadas por procesos de remoción en masa, 683 personas se localizan en cerro La Virgen y 458 en cerro Corcovado II.

\section{EXPOSICIÓN A AMENAZA POR ANEGAMIENTO}

La amenaza por procesos de anegamiento, asociado a la saturación de las napas freáticas producto de las precipitaciones (Mardones y Vidal, 2001), es el que presenta la menor cantidad de población expuesta; 4.633 personas, equivalentes al 5,3\% los que se distribuyen en seis distritos: Villa Mora, Schwager, Buen Retiro, Corcovado, Playa Negra y Escuadrón.

El distrito censal de Schwager es el que presenta el más alto número de personas expuestas frente a la amenaza de anegamiento, con 1.941 personas (12,3\%), de estas 1.110 se ubican en el sector del estero Maule y 831 personas se emplazan en el sector La Obra (Población Nuevo Amanecer). En Villa Mora existen 1.606 personas (10,6\%) ubicadas en áreas de amenaza, 1.381 de ellas, pertenecen al sector del estero Villa Mora, mientras que 106 habitantes a la planicie de Calabozo. En el distrito de Corcovado 105 personas están expuestas, lo que corresponde a un 9,9\% de la población del distrito. En el distrito de Buen Retiro, 118 personas $(0,73 \%)$ son las expuestas a esta amenaza, ubicadas cercana al estero Maule. El distrito de Escuadrón presenta 107 personas expuestas a esta amenaza, las que están situadas principalmente en la llanura de Lagunillas, y también en el sector del estero Villa Mora. Finalmente en el distrito censal de Playa Negra, el 100\% de sus habitantes está expuesto a esta amenaza (28 personas). 


\section{EXPOSICIÓN A LA AMENAZA DE INUNDACIÓN POR TSUNAMI}

La inundación por tsunami constituye una de los procesos que ocupan una mayor dimensión en cuanto a superficie afectada $\left(12,23 \mathrm{~km}^{2}\right)$ y 5.208 personas expuestas a esta amenaza. El distrito censal Escuadrón es el que tiene mayor población expuesta a la inundación por tsunami con 1.781 habitantes expuestos (5,7\%); luego el distrito de Municipalidad con 1.246 personas habitando áreas expuestas a esta amenaza constituyen un 25\% de la población del distrito; así también alcanza un nivel de exposición relevante, el distrito de Corcovado con 1.288 personas expuestas que representan un 15,4\% de la población del distrito; con menores porcentajes de población expuesta a tsunami están los distritos de Schwager con 392 personas (2,5\%); Buen Retiro con 241 personas y Villa Mora 231 personas habitando estas zonas estas últimas representan un 1,5\% de la población de estos distritos.

\section{EXPOSICIÓN RIESGOS INTEGRADOS}

Del análisis de las cartas de amenazas (figura 2) y exposición (figura 4), se puede indicar en forma decreciente que la amenaza más significativa considerando el número de personas expuestas
(11.354 habitantes) es la de remoción en masa, seguida de la amenaza de inundación por tsunami, a la cual se encuentran expuestas 5.208 personas. Luego nos encontramos con la amenaza de subsidencia, que afecta a 5.080 personas, este riesgo es de cuidado, dado que su ocurrencia es impredecible ya que, generalmente, no hay fenómeno que lo anticipe. La población ubicada en áreas de amenaza de remoción en masa y subsidencia, corresponde a sectores antiguos de la ciudad, de formación irregular y espontánea (autoconstrucción y tomas de terreno). La zona con menor número de personas expuestas, corresponde a nuevas edificaciones en la ciudad, al norte del estero Villa Mora. Estas son poblaciones que pertenecen a proyectos inmobiliarios o viviendas sociales. La amenaza por anegamiento, tiene una menor presencia en la ciudad, esta afectaría a un total de 3.475 personas, ubicadas en la localidad del estero Maule (1.228), localidad del estero Villa Mora (1.416) y en el sector La Obra (831).

Finalmente, existen distritos que presentan población expuesta a diversas amenazas, transformándose en áreas urbanas de mayor relevancia, que deben ser consideradas en los procesos de actualización de los Planes Reguladores Comunales y de enfoques de gestión del riesgo y atención a las emergencias (tabla 3). 


\section{TABLA 3. DISTRIBUCIÓN DE LA POBLACIÓN EXPUESTA POR TIPOLOGÍA DE AMENAZA A NIVEL DE DISTRITO}

\begin{tabular}{lrrrr|}
\hline \multirow{2}{*}{$\begin{array}{l}\text { Distrito } \\
\text { Censal }\end{array}$} & \multicolumn{3}{c|}{ Población expuesta (nro. habitantes) } \\
\cline { 2 - 5 } & Remoción en masa & Subsidencia & Anegamiento & $\begin{array}{r}\text { Inundación por } \\
\text { tsunami }\end{array}$ \\
Villa Mora & 4.031 & 240 & 1.606 & 231 \\
\hline Schwager & 5.638 & 3.699 & 1.941 & 392 \\
\hline Corcovado & 1.685 & 1.141 & 833 & 1288 \\
\hline Buen retiro & - & - & 118 & 241 \\
\hline Playa negra & - & - & 28 & 28 \\
\hline Escuadrón & - & - & 107 & 1781 \\
\hline Totales & 11.354 & 5.080 & 4.633 & 5.208
\end{tabular}

Fuente: Elaboración propia, a partir de los análisis de los autores según tipología de amenaza.

\section{Conclusiones}

Conocer las poblaciones que serían más afectadas en caso de la ocurrencia de algún desastre natural, sirve para desarrollar una gestión proactiva del riesgo, ya que se tiene una visión territorial compacta de las características de las zonas expuestas. Este hecho, permitiría desarrollar tanto medidas preventivas como acciones estructurales y de ordenación del territorio, disminuyendo la vulnerabilidad ante el riesgo.

La carta de amenaza generada por el Plan Regulador Comunal de Coronel, entrega información que es discutible y que el expediente urbano no clarifica. Por ejemplo, para el riesgo de inundación solo se determinan puntos de desborde de los esteros y no las zonas que afectaría este. Asimismo para el riesgo de marejadas, se establecen los lugares donde estas ocurren, pero no la superficie involucrada. En relación con el riesgo de remoción en masa, determina las áreas de deslizamientos, pero no marca como superficie de riesgo las zonas de acumulación de material, las que serían igualmente afectadas. Tomando en consideración lo antes mencionado, la carta de peligrosidad debiera modificarse y, por lo tanto, los resultados obtenidos de exposición de la población serían considerablemente distintos.

Del análisis de la carta de amenazas y exposición, se puede ver que el fenómeno natural más 
significativo en superficie afectada es la amenaza por inundación de tsunami que se expresa en $12,33 \mathrm{~km}^{2}$ en las llanuras costeras de la ciudad de Coronel; en esta zona se localiza más de 5.208 habitantes. Sin embargo, la amenaza de remoción en masa (con $6,8 \mathrm{~km}^{2}$ y 11.354 habitantes, respectivamente) es la que contemplaría un mayor número de personas expuestas; el fenómeno de subsidencia, es de igual modo importante tomando en cuenta la cantidad de personas expuestas a esta amenaza (5.080 personas). La amenaza por subsidencia generalmente no tiene un fenómeno que lo anticipe, a diferencia de la remoción en masa y anegamiento. De menor relevancia es la amenaza por anegamiento fenómeno al que se encuentran expuestas 4.633 personas.

Dada la cantidad de población expuesta a amenazas naturales, se pueden reconocer áreas de posición jerarquizada, de esta manera se pueden orientar las acciones municipales, en relación con los riesgos naturales, hacia los sectores que las requieran, que son los que presentan mayor exposición al riesgo.

Para Coronel y para todas las comunas, sería necesario hacer un estudio integrado de riesgos que abarque los tres factores del riesgo: amenaza, vulnerabilidad y exposición, puesto que el Estudio Fundado de Riesgos de los instrumentos de planificación territorial, en particular de los Planes Reguladores Comunales (PRC), consideran solo el factor amenaza. Un estudio integrado permitirá tener una visión completa de espacialización de las amenazas naturales y antrópicas, los niveles de vulnerabilidad de las comunidades expuestas a esas amenazas, transformándose así en un instrumento de gestión y prevención que se focalice en zonas vulnerables, como podría ser elaborar un programa de mejoramiento de estos barrios, que contenga la ejecución de medidas tanto estructurales como no estructurales, tendientes a disminuir su vulnerabilidad.

Finalmente, se indica que aquellos organismos que generen información territorial, deben trabajar en sistemas compatibles, con información debidamente procesada y georreferenciada. Para este caso, la información de población proporcionada por el Instituto Nacional de Estadísticas presentó diferencias en su georreferenciación, lo que afecta la precisión y exactitud, tanto de la cobertura como de los datos. Cabe precisar que los resultados de este estudio se basan en el censo de población 2002, lo que implica que en los 14 años posteriores ha habido un aumento de la población, lo que podría generar diferencias en el número y porcentaje de población expuesta a las amenazas naturales. 


\section{Bibliografía}

Aliste, E., Contreras, M. y Sandoval, V. (2012). Industrialización, desarrollo y ciudad: transformaciones socio-demográficas y espaciales en la geografía social del gran Concepción (19502010). Revista INVI, 27(75), 21-71. doi:10.4067/ S0718-83582012000200002

Ashly, R. (2010). Escenario planning. Manuscrito no publicado. Texto de estudio en curso intensivo de formación "Integrated Flood Risk Management". UNESCO-IHE. Delft.

Ayala, F. y Olcina, J. (2002). Riesgos naturales. Barcelona: Ariel.

Bevilacqua, M., Ciarapica, F. y Paciarotti, C. (2014). A BPR approach to hydrogeological risk management. Natural Hazards, 71(3), 1995-2012. doi:10.1007/s11069-013-0993-3

Boisier, S. (2001). Desarrollo local: ¿De qué estamos hablando? Transformaciones globales. Instituciones y políticas de desarrollo local (pp. 48-74). Rosario: Homo Sapiens.

Burgos, M. y Muñoz, M. (2007). Geografía. Ciencias sociales. $3^{\circ}$ Educación secundaria. Madrid: Anaya.

Fuentes, P. Y. P., Leonel. (2012). Formación del Concepción metropolitano a través de los grandes conjuntos residenciales: Aportaciones del urbanismo moderno. Atenea(505), 33-78. doi:10.4067/ S0718-04622012000100003
Llanos-Hernández, L. (2015). El concepto del territorio y la investigación en las ciencias sociales. Agricultura, Sociedad y Desarrollo, 7(3), 207-220.

Mardones, M. y Vidal, C. (2001). La zonificación y evaluación de los riesgos naturales de tipo geomorfológico: un instrumento para la planificación urbana en la ciudad de Concepción. EURE Revista Latinoamericana de Estudios Urbano Regionales, 27(81), 97-122. doi:10.4067/S0250-71612001008100006

Martí, C. (2005). La transformació del paisatge litoral de la Costa Brava. Anàlisi de l'evolució (1956-2003), diagnosi de l'estat actual i prognosi de futur. (Tesis doctoral), Universidad de Girona, Girona. Recuperado de http://www.tdx.cat/handle/10803/7899

Mendoza, R. (2000). El género y los enfoques de desarrollo. Escuela para el Desarrollo

Muñiz, I., Sánchez, V. y García-López, M.-Á. (2015). Estructura espacial y densidad de población en la ZMVM 1995-2010: evolución de un sistema urbano policéntrico. EURE Revista Latinoamericana de Estudios Urbano Regionales, 41(122), 75-102. doi:10.4067/S0250-71612015000100004

ONEMI. (2006). Estadísticas de impacto en personas y viviendas por sistemas frontales en la VIII Región, periodo 2000-2006. Santiago: Oficina Nacional de Emergencias del Ministerio del Interior (ONEMI), Departamento de Protección Civil.

Pujadas, R. y Font, J. (1998). Ordenación y planificación territorial. Madrid: Síntesis.

Quezada, J., Jaque, E., Fernández, A. y Vásquez, D. (2012). Cambios en el relieve generados como 
consecuencia del terremoto $\mathrm{Mw}=8,8 \mathrm{del} 27 \mathrm{de}$ febrero de 2010 en el centro-sur de Chile. Revista de Geografía Norte Grande(53), 35-55. doi:10.4067/ S0718-34022012000300003

Rojas, C., Muñiz, I. y Pino, J. (2013). Understanding the Urban Sprawl in the Mid-Size Latin American Cities through the Urban Form: Analysis of the Concepción Metropolitan Area (Chile). Journal of Geographic Information System(5), 222-234. doi:10.4236/jgis.2013.53021

Serra, A. (2011). Turning hazards into resources? floods, wetlands and climate change in Mediterranean coast of Spain. (Tesis doctoral ), Universidad Autónoma de Barcelona, Barcelona.

Silva, G. y Romero, M. (2015). Sustentabilidade urbana aplicada: Análise dos processos de dispersão, densidade e uso e ocupação do solo para a cidade de Cuiabá, Estado de Mato Grosso, Brasil. EURE Revista Latinoamericana de Estudios Urbano Regionales, 16(122), 209-237. doi:10.4067/ S0250-71612015000100010

Smith, P. y Romero, H. (2009). Efectos del crecimiento urbano del Área Metropolitana de Concepción sobre los humedales de Rocuant-Andalién, Los Batros y Lenga. Revista de Geografía Norte Grande(43), 81-93. doi:10.4067/S0718-34022009000200005

Suazo, B., Jaque, E., Rojas, C. y Utz, R. (2009). Assessment of sustainability in urban planning of a coastal city: the city of Coronel, southern Chile. En C. Brebbia, M. Neophytou, E. Beriatos, I. Ioannou y A. Kungolos (Eds.), Sustainable Development and Planning IV (Vol. 2, pp. 665-678). Southampton: WIT Press.

SUBDERE. (2011). Plan regional de ordenamiento territorial: contenido y procedimientos. Santiago: Autor.

United Nations International Strategy for Disaster Reduction. (2009). Terminología sobre reducción del riesgo de desastre. Recuperado de http://www. unisdr.org/files/7817_UNISDRTerminologySpanish.pdf

Wilches-Chaux, G. (1993). La vulnerabilidad global. En Los Desastres no son naturales (pp. 11-44): La Red.

Zilbert, L. (2010). Evolución de las políticas de reducción de riesgo de desastres. En PNUD (Ed.), Diplomado de Especialización en Desarrollo Local y Gestión Integral del Riesgo (hoja de ruta) PNUD: escuela virtual. 